\title{
EFFECT OF A NITROGEN IMPURITY ON THE FUNDAMENTAL RAMAN BAND OF DIAMOND SINGLE CRYSTALS
}

\author{
G. A. Gusakov, ${ }^{a *}$ M. P. Samtsov, ${ }^{a}$ and E. S. Voropay ${ }^{b}$
}

UDC 535.375.5:548.736.15

The effect of nitrogen defects in natural and synthetic diamond single crystals on the position and half-width of the fundamental Raman band was investigated. Samples containing the main types of nitrogen lattice defects at impurity contents of 1-1500 ppm were studied. The parameters of the Stokes and anti-Stokes components in Raman spectra of crystals situated in a cell with distilled water to minimize the influence of heating by the exciting laser radiation were analyzed to determine the effect of a nitrogen impurity in the diamond crystal lattice. It was shown that an increase of impurity atoms in the crystals in the studied concentration range resulted in broadening of the Raman band from 1.61 to $2.85 \mathrm{~cm}^{-1}$ and shifting of the maximum to lower frequency from 1332.65 to $1332.3 \mathrm{~cm}^{-1}$. The observed effect was directly proportional to the impurity concentration and depended on the form of the impurity incorporated into the diamond lattice. It was found that the changes in the position and half-width of the fundamental Raman band for diamond were consistent with the magnitude of crystal lattice distortions due to the presence of impurity defects and obeyed the Gruneisen law.

Keywords: diamond single crystal, Raman scattering, sample temperature, impurity.

Introduction. Diamonds have great potential in instrumentation because they combine unique optical, electronic, thermal, and mechanical properties with high radiation and chemical resistance. The advantages of diamond over the traditional materials of the electronics industry such as silicon and gallium arsenide were recognized long ago [1, 2]. However, a lack of the required amount of natural raw material of the required quality and its high cost delayed for a long time the development of electronics based on diamonds. Interest in diamonds for electronics has recently risen considerably because of advancements in diamond synthesis technology, primarily homoepitaxial growth from the gas phase of single-crystalline layers of high-purity semiconducting diamond on diamond substrates (CVD process) [3, 4]. This technology already allows single-crystalline diamond layers to be grown on an area of several dozen square centimeters and to be doped during growth. Problems with fabricating spacious high-quality diamond substrates must be solved before semiconducting CVD-diamond synthesis technology can develop further. Also, nondestructive diagnostic methods for the condition of such substrates and the grown epitaxial diamond layers are needed.

Raman spectroscopy is one of the most informative methods for elucidating the structure and phase composition of carbon materials. The first-order Raman spectrum (RS) of diamond single crystal is a single narrow line resulting from light scattering at a triply degenerate $T O(X)$ phonon from the center of the Brillouin zone. The line maximum occurs at $1332.5 \pm 0.5 \mathrm{~cm}^{-1}$ and has a half-width of $\sim 1.7 \mathrm{~cm}^{-1}$ for structurally perfect crystals at room temperature [5]. The position and half-width of this line can be affected by various factors such as temperature, external pressure, internal strains, the sample isotopic and impurity composition, etc. [5,6]. Thus, the characteristics of the basic line in the diamond RS can provide a measure of the sample structural perfection and also be used for measuring the level of external effects on the sample. Effects of dopants on the diamond RS parameters is currently little discussed in the literature despite the fact that the physical properties of diamond are determined mainly by the impurity composition. Boron (B) has been the subject of most investigations of dopants because its presence can produce $p$-type semiconducting diamonds. For example, the basic line in the low-frequency RS of homoepitaxial CVD-diamond films doped with B was shown to shift to lower frequency as the content of the dopant in the diamond lattice increased [7-9]. This shift was small with low B concentrations but reached

*To whom correspondence should be addressed.

${ }^{\mathrm{a}}$ A. N. Sevchenko Institute of Applied Physical Problems, 7 Kurchatov Str., Minsk, 220045, Belarus; email: gga68@rambler.ru; ${ }^{b}$ Belarusian State University, Minsk, Belarus. Translated from Zhurnal Prikladnoi Spektroskopii, Vol. 85, No. 2, pp. 262-270, March-April, 2018. Original article submitted December 18, 2017. 
$10-12 \mathrm{~cm}^{-1}$ for samples with dopant content $\sim 2 \cdot 10^{20} \mathrm{~cm}^{-3}[7,8]$. The line was also observed to be noticeably broadened. Increasing the $\mathrm{B}$ concentration further caused a sharp low-frequency shift of the line position to $1283 \mathrm{~cm}^{-1}$ at concentrations of $\sim 10^{21} \mathrm{~cm}^{-3}$ [9]. Asymmetry due to Fano resonance was observed in the RS basic line at high B dopant concentrations. Furthermore, two additional bands with maxima near 500 and $1230 \mathrm{~cm}^{-1}$ appeared in the RS [6-9]. The nature of these bands has not been finally established. It was proposed that the band at $500 \mathrm{~cm}^{-1}$ corresponded to a pair of $\mathrm{B}$ atoms at neighboring lattice sites [10] and that the exact position of the maximum of band Lorentzian component could be used to determine the diamond doping level.

The effects of nitrogen $(\mathrm{N})$ on the RS parameters of diamond have been much less elaborated although it is the principal impurity of both natural and synthetic diamond single crystals. Several researchers cited an early publication [11] to claim that $\mathrm{N}$ dopant did not affect the position of the diamond RS line maximum. This was confirmed in several later experimental studies $[12,13]$ where the line position was not observed to shift within experimental accuracy if the content of $\mathrm{N}$ dopant was increased in the range $5 \cdot 10^{17}-5 \cdot 10^{19} \mathrm{~cm}^{-3}(3-300 \mathrm{ppm})$. It was also found that the basic-line half-width increased linearly as the $\mathrm{N}$ concentration increased. The line broadening was $20-30 \%$ for the highest studied $\mathrm{N}$ concentration. RS of synthetic diamond single crystals containing $\mathrm{N}\left(2 \cdot 10^{17}-1.4 \cdot 10^{20} \mathrm{~cm}^{-3}\right)$ were studied [14]. The crystals contained impurities as solitary atoms ( $C$-defect) and as complexes $(A$-defect). A linear dependence was demonstrated for the impurity content and the RS line width. Its slope differed for different types of $\mathrm{N}$ defects. Furthermore, the observed effect was explained qualitatively by comparing diamond lattice defects caused by the different $\mathrm{N}$ defects. The position of the RS basic line changed systematically with $\mathrm{N}$ concentration in diamonds with a maximum shift of $0.5-0.7 \mathrm{~cm}^{-1}$ [14]. However, this effect was not studied in detail because of the experimental uncertainty of $0.3 \mathrm{~cm}^{-1}$ in the position of the line maximum [14]. The position of the RS basic line shifted by $1-2 \mathrm{~cm}^{-1}$ in highly N-doped $\left(\sim 2 \cdot 10^{20} \mathrm{~cm}^{-3}\right)$ synthetic diamond powders produced by spontaneous crystallization [15]. Later, a shift to lower frequencies by $2.5-3.0 \mathrm{~cm}^{-1}$ of the RS basic line maximum at a $\mathrm{N}$ doping level of $\sim 2 \cdot 10^{20} \mathrm{~cm}^{-3}$ was also observed for synthetic diamond single crystals grown on a seed [16]. Thus, the presented literature suggested that the $\mathrm{N}$ impurity had a much smaller effect on the RS of diamond than $\mathrm{B}$. The characteristic changes in the position of the RS basic line varied from tenths to units $\mathrm{of}^{-1}$ as the $\mathrm{N}$-dopant concentration increased. Raman spectroscopic studies of such small effects are an extremely challenging problem. An analysis of the results must consider the effects of other factors, e.g., internal strains caused by an uneven dopant distribution or other structural defects, local heating of the crystal by the analytical laser beam, etc. The effects of the various factors are also problematical to separate correctly. Apparently, this is responsible for the few publications on the effects of dopants on diamond RS parameters and the considerable scatter of the results.

Previously, we showed that diamond samples could be heated by several dozen degrees for the typical range of laserradiation power $70-480 \mathrm{~mW}\left(0.9-6.0 \mathrm{~kW} / \mathrm{cm}^{2}\right)$ that, as a rule, is used to excite diamond RS [17]. This shifted the diamond line maximum by $\sim 0.5 \mathrm{~cm}^{-1}$ and increased the RS band half-width of single crystals to $\sim 0.15 \mathrm{~cm}^{-1}$. The effect of sample heating by the laser beam on the parameters of the diamond RS basic line was comparable or even greater than the effect of dopants for crystals with high absorption coefficients at the laser-radiation analytical wavelength. Procedures for taking measurements and processing their results that enabled effects of temperature and dopants on the parameters of the RS basic line of single crystals were also proposed [17].

The goal of the present work was to find the effect of a N impurity on the RS parameters by studying a series of single crystalline samples of both natural and synthetic diamond with various $\mathrm{N}$ contents using the literature method [17].

Sample Characteristics. A total of 14 samples of diamond single crystals (10 synthetic and 4 natural diamond samples) were studied (Table 1). Single crystals of synthetic diamond were grown via the temperature-gradient method using a high-pressure apparatus of the split-sphere type (HASS technology) $[18,19]$. The impurity composition of the samples was varied by intentionally changing the crystallization conditions. A set of standard samples for the UKT-3 system for measuring diamond thermal conductivity by exploring heat transport across a specimen were used as the samples of natural diamond with different $\mathrm{N}$ contents [20]. The thermal conductivity of the standard samples varied over the broad range 400-1800 $\mathrm{W} / \mathrm{m} \cdot \mathrm{K}$. A considerable variation of the impurity contents in these samples was expected because the thermal conductivity of diamond crystals is known to depend significantly on the $\mathrm{N}$ content in them [5].

The studied samples were plane-parallel plates cut in the (111) plane of thickness $0.4-1.1 \mathrm{~mm}$ and linear dimensions from $2 \times 3$ to $3 \times 4 \mathrm{~mm}$. The internal strain level was monitored by analyzing birefringence using an R-113 polarizing microscope (LOMO). All samples were relatively uniformly gray in crossed polarizers. This indicated a lack in them of noticeable internal strain. The impurity compositions of the samples were determined by analyzing absorption spectra in the range $0.2-25.0 \mu \mathrm{m}$ using intensities of bands corresponding to impurity defects in the diamond lattice and published 

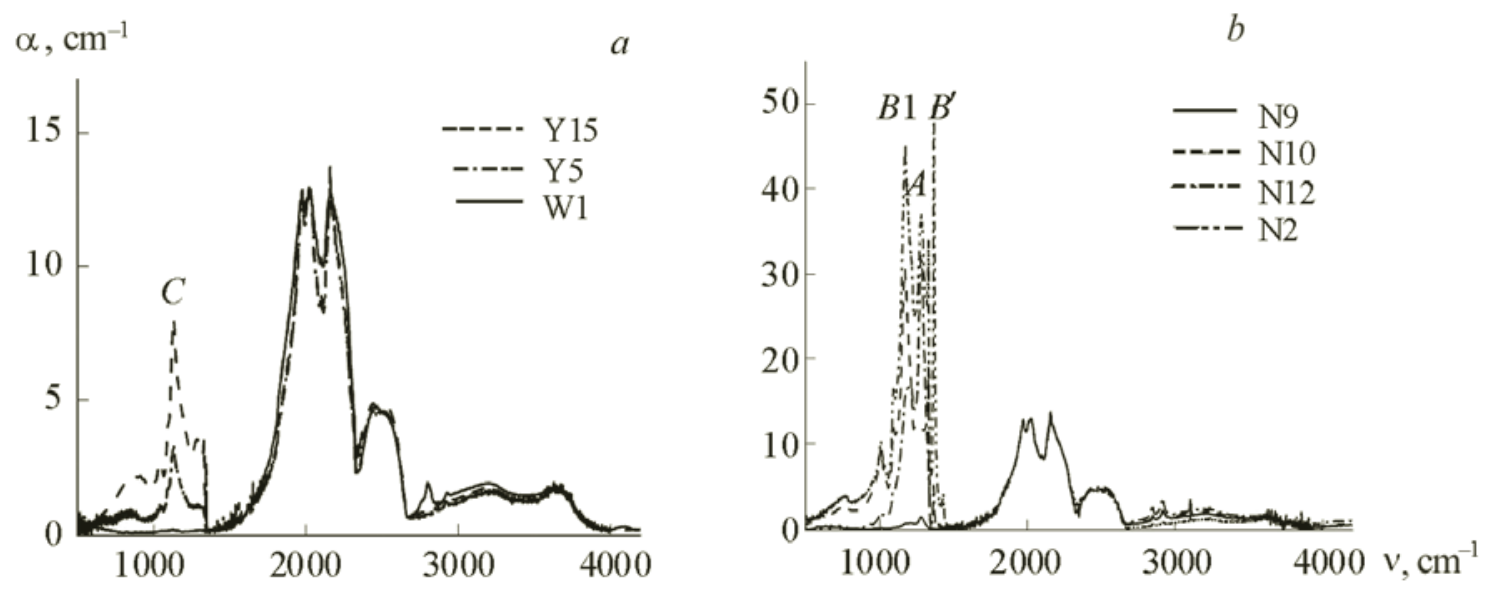

Fig. 1. IR absorption spectra of synthetic (a) and natural diamond (b).

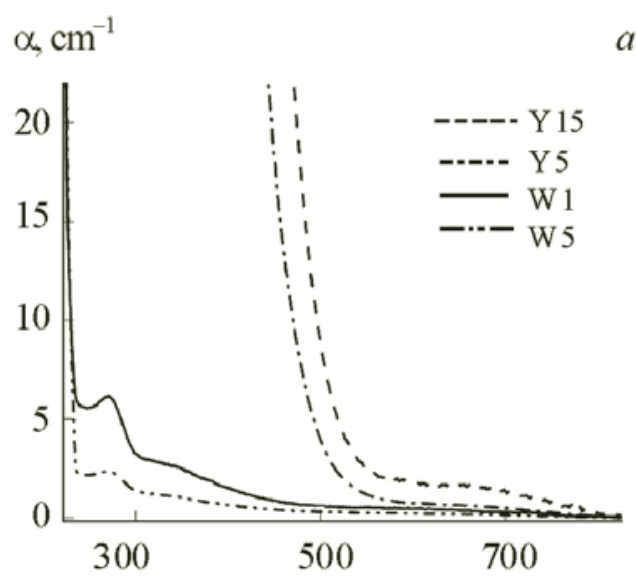

$a$

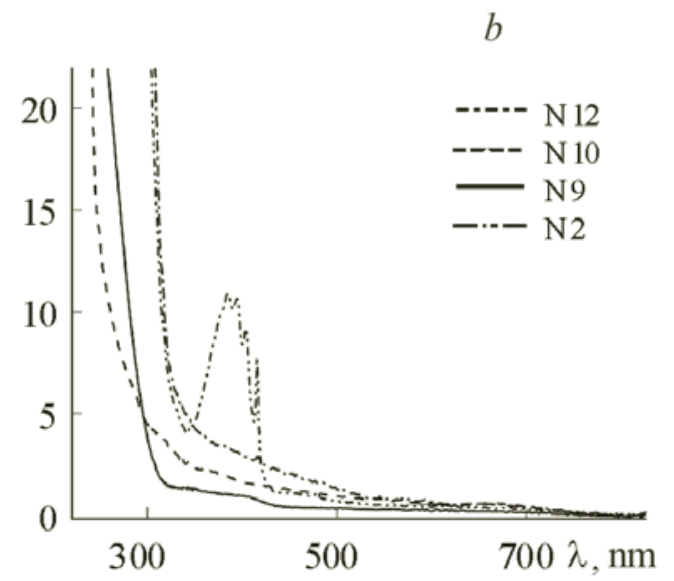

Fig. 2. UV and visible absorption spectra of synthetic (a) and natural diamond (b).

proportionality coefficients [5]. The impurity concentration was expressed in units of ppm (number of impurity atoms per million lattice atoms). Absorption spectra in the range $0.2-0.9 \mu \mathrm{m}$ were recorded using a Cary 300 spectrophotometer (Varian); IR absorption spectra, a Vertex 70 FTIR (Bruker).

Figures 1 and 2 and Tables 1 and 2 present the optical characteristics of the samples. Broad bands in the range $1500-3500 \mathrm{~cm}^{-1}$ for intrinsic vibrations of the diamond lattice atoms were recorded for all studied crystals in the IR absorption spectra (Fig. 1). Furthermore, most samples exhibited a group of bands in the range $1000-1400 \mathrm{~cm}^{-1}$ that were due to vibrational modes of $\mathrm{N}$ dopant in various defects [5]. A band at $1130 \mathrm{~cm}^{-1}$ dominated the range $1000-1400 \mathrm{~cm}^{-1}$ for all synthetic diamonds and was associated with single $\mathrm{N}$ atoms in substitution positions ( $C$-defects) [5]. Thus, these samples could be assigned to the pure Ib type. Synthetic diamonds W1 and W5 with low N contents absorbed weakly in the range $1000-1400 \mathrm{~cm}^{-1}$ so that the impurity concentration could not be correctly determined using IR absorption spectra. For these samples, the $\mathrm{N}$ concentration was determined from the UV absorption intensity at $270 \mathrm{~nm}$ (Fig. 2a), which was associated with $\mathrm{N}$ dopant as $C$-defects [5]. Table 1 shows that the $\mathrm{N}$-dopant concentration in the synthetic diamond samples varied in the range 1-200 ppm.

Figure $1 \mathrm{~b}$ shows IR absorption spectra of natural diamond samples N2, N9, N10, and N12. A band at $1280 \mathrm{~cm}^{-1}$ for $A$-defects (pair of $\mathrm{N}$ atoms in neighboring lattice sites) dominated the range $1000-1400 \mathrm{~cm}^{-1}$ for samples N9 and N12 [5]. $A$ band at $1175 \mathrm{~cm}^{-1}$ for $B 1$ defects (four $\mathrm{N}$ atoms in the first coordination sphere of a vacancy) dominated the same range for N2 and N10 [5]. Furthermore, a strong absorption band at $1365 \mathrm{~cm}^{-1}$ corresponding to $B^{\prime}$ defects was observed for N2. 
TABLE 1. Impurity Contents in Studied Samples

\begin{tabular}{|c|c|c|c|c|c|}
\hline \multirow{2}{*}{ Sample } & \multirow{2}{*}{$\begin{array}{c}\text { Total impurity } \\
\text { content, ppm }\end{array}$} & \multicolumn{3}{|c|}{ N impurity content, ppm } & \multirow{2}{*}{$\begin{array}{c}B^{\prime} \text { defect content } \\
\left(\alpha_{1365}, \mathrm{~cm}^{-1}\right)\end{array}$} \\
\cline { 3 - 6 } & 3 & as C-defects & as $A$-defects & as B1-defects & - \\
W1 & 1 & 3 & - & - & - \\
W5 & 78 & 78 & - & - & - \\
Y5 & 129 & 129 & - & - & - \\
Y6 & 190 & 190 & - & - & - \\
Y7 & 146 & 146 & - & - & - \\
Y8 & 176 & 176 & - & - & - \\
Y9 & 165 & 165 & - & - & - \\
Y10 & 200 & 200 & - & - & - \\
Y15 & 118 & 118 & - & - & 48.5 \\
Y21 & 1502 & - & - & 1207 & - \\
N2 & 27 & - & 295 & 3 & - \\
N9 & 1074 & - & 6 & 1068 & 5.2 \\
N10 & 627 & - & 494 & 133 & \\
N12 & & & & & \\
\hline
\end{tabular}

TABLE 2. Optical Characteristics of Studied Samples

\begin{tabular}{|c|c|c|c|c|}
\hline Sample & Sample thickness, $\mathrm{mm}$ & $\alpha_{481.5 \mathrm{~nm}}, \mathrm{~cm}^{-1}$ & $\alpha_{514.5 \mathrm{~nm}}, \mathrm{~cm}^{-1}$ & $\alpha_{522.4 \mathrm{~nm}}, \mathrm{~cm}^{-1}$ \\
\hline W1 & 1.1 & 0.87 & 0.75 & 0.7 \\
W5 & 1.0 & 0.75 & 0.7 & 0.7 \\
Y5 & 0.75 & 7.2 & 2.6 & 2.1 \\
Y6 & 0.7 & 12.9 & 5 & 2.3 \\
Y7 & 0.5 & 16.5 & 6.3 & 2.45 \\
Y8 & 0.4 & 11.9 & 4.7 & 2.4 \\
Y9 & 0.7 & 14.0 & 5.7 & 2.9 \\
Y10 & 0.55 & 14.2 & 5.8 & 4.4 \\
Y15 & 0.4 & 14.9 & 5.9 & 2.4 \\
Y21 & 0.65 & 11.1 & 3.9 & 1.7 \\
N2 & 0.8 & 0.95 & 0.7 & 0.55 \\
N9 & 0.5 & 0.45 & 0.4 & 0.35 \\
N10 & 0.45 & 1.1 & 1 & 0.8 \\
N12 & 0.3 & 1.6 & 1.1 & 0.85 \\
\hline
\end{tabular}

These defects were non-nitrogen in nature according to the last data [5]. In general, all studied natural diamond samples could be regarded as mixed type Ia according to how the $\mathrm{N}$-dopant was incorporated into the crystal lattice. The total content in them of N-dopant varied from 27 to $1500 \mathrm{ppm}$.

RS Measurement Method. RS were recorded at room temperature using a Model 1403 Raman spectrometer equipped with a double monochromator (Spex) and an R928 photomultiplier cooled to $243 \mathrm{~K}$. The spectrometer resolution in the spectral range $\sim 500 \mathrm{~nm}$ was $0.15 \mathrm{~cm}^{-1}$. Spectra were recorded in reverse scattering geometry with excitation by $\mathrm{Ar}^{+}$-laser radiation with $\lambda=514.5 \mathrm{~nm}$. The exciting beam was focused on the sample surface into a spot $\sim 100 \mu \mathrm{m}$ in diameter. The power of the laser radiation was recorded by an LM2 power meter and was varied in the range 400 $70 \mathrm{~mW}$ by inserting NS neutral light filters. The average power density in the laser spot varied from 89 to $611 \mathrm{~kW} / \mathrm{cm}^{2}$. 

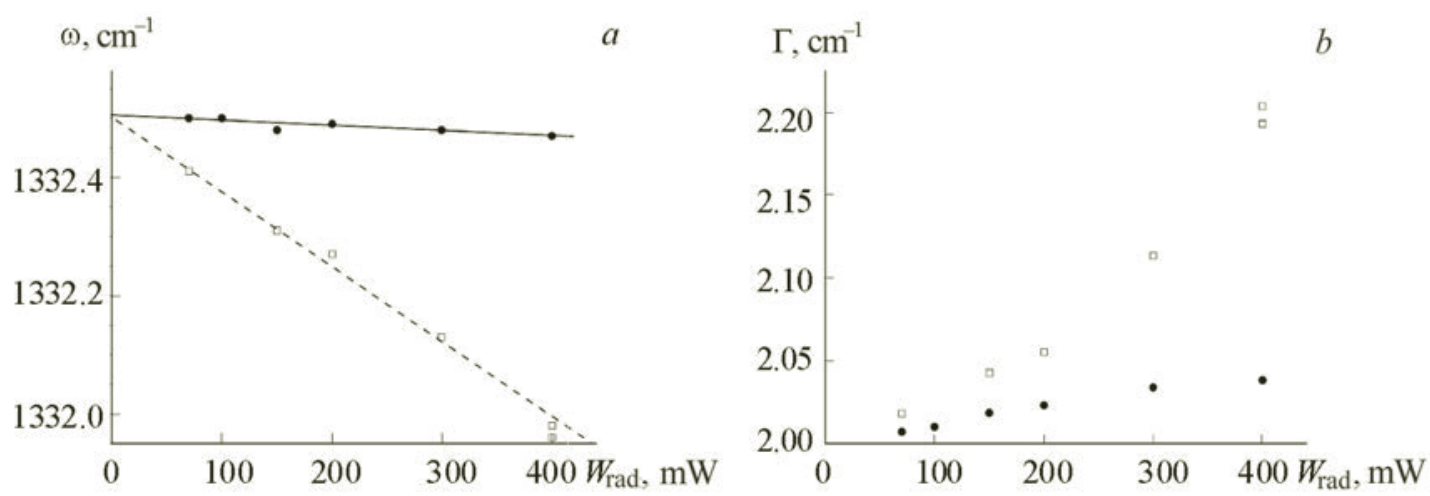

Fig. 3. Position of maximum (a) and half-width (b) of the diamond Raman line as functions of exciting laser radiation power for sample Y15 lying freely on the spectrometer sample stage (口) and in a cuvette with $\mathrm{H}_{2} \mathrm{O}(\bullet)$.

The spectral half-width of the spectrometer slits was set at $0.25 \mathrm{~cm}^{-1}$. A scrambler was inserted before the spectrometer input slit to avoid polarization effects of the recorded radiation. The spectral sensitivity of the recording monochromator and photomultiplier system was calibrated using the standard method [21] and standard lamps, i.e., a Q6.6A/T4QIICL 200-W tungsten halogen lamp (220 A model) and an SI6-40 tungsten lamp (at brightness temperatures 2000 and $2400 \mathrm{~K}$ ). All measurements were made under normal atmospheric conditions at 293-295 K. The literature method based on sequential recording of Stokes and anti-Stokes RS components was used to determine the exact position of the diamond RS line [17]. The parameters of the recorded diamond RS lines were found by approximating the experimental data by a Lorentz function using Origin 7 software. This approach could determine the position of the diamond RS band maximum to an accuracy of $0.05 \mathrm{~cm}^{-1}$ or better.

The principal measurements were taken at the minimal laser-radiation (LR) power to reduce sample heating by the laser beam. This enabled the anti-Stokes component of the diamond RS to be consistently recorded (this power was $70 \mathrm{~mW}$ under our experimental conditions). The sample temperature during the measurements was monitored as before [17] by comparing the intensities of the Stokes and anti-Stokes RS components considering the actual sample absorption spectrum. Table 2 presents the optical characteristics of the samples that were necessary for this.

Results and Discussion. The research showed that diamond plates lying freely on the spectrometer duraluminum sample stage were heated insignificantly $(1-2 \mathrm{~K})$ by the laser beam if they had low absorption coefficients at the wavelength of the exciting LR $\left(\alpha_{514} \mathrm{~nm} \leq 1 \mathrm{~cm}^{-1}\right)$. However, crystals with high absorption coefficients at the wavelength of the exciting LR $\left(\alpha_{514 \mathrm{~nm}}>4 \mathrm{~cm}^{-1}\right)$ were heated up to $10-15 \mathrm{~K}$ [17]. This shifted the RS line maximum by $0.1-0.15 \mathrm{~cm}^{-1}$ and could seriously hinder the analysis of the effects of impurities in the diamond lattice. The studied crystals were placed into a quartz cuvette with distilled $\mathrm{H}_{2} \mathrm{O}$ during the measurements to improve heat transfer from the samples. An analogous approach was used before [22]. Figure 3 shows the effectiveness of this methodical approach for sample Y15. The RS line maximum shifted to lower frequency as the LR power grew if the crystal lay freely on the spectrometer sample stage, indicating the sample was heated significantly by the laser beam. If the sample was placed into a cuvette with $\mathrm{H}_{2} \mathrm{O}$, then the position of the line maximum was practically constant as the LR power grew. A similar effect occurred for the line half-width (Fig. 3b).

Figure 4 shows the dependences of the position of the maximum and half-width of the RS basic line on the N-dopant content in various diamond single crystals. It can be seen (Fig. 4a) that the parameters of the RS basic lines were the same for natural and synthetic diamonds with low impurity contents. The positions of the line maximum $\omega$ for these samples at $294 \mathrm{~K}$ fell in the range $1332.6-1332.65 \mathrm{~cm}^{-1}$; line half-width $\Gamma, \sim 1.6 \mathrm{~cm}^{-1}$. The line maximum shifted to lower frequency for all studied samples as the N-dopant content in the crystals increased. However, the slope of the function for the position of the RS basic line maximum vs. the N-dopant content, $\omega(\mathrm{N})$, was greater for synthetic diamonds than for natural ones. The maximum line shift $\Delta \omega \sim 0.35 \mathrm{~cm}^{-1}$ for natural diamonds was observed with an N-dopant content of $\sim 1500 \mathrm{ppm}$. The dependence of the line maximum position on the $\mathrm{N}$-dopant content for synthetic diamonds had a considerably greater slope. The maximum line shift $\Delta \omega \sim 0.15 \mathrm{~cm}^{-1}$ was observed for an $\mathrm{N}$-dopant content of $200 \mathrm{ppm}$. An analogous picture was seen for the half-width of the diamond RS basic line. The line half-width $\Gamma$ increased for all studied samples as the $\mathrm{N}$-dopant content increased. Like for the position of the line maximum, the slope of the dependence of the RS basic line half-width on the $\mathrm{N}$-dopant content, $\Gamma(\mathrm{N})$, was greater for synthetic diamonds than for natural ones. 

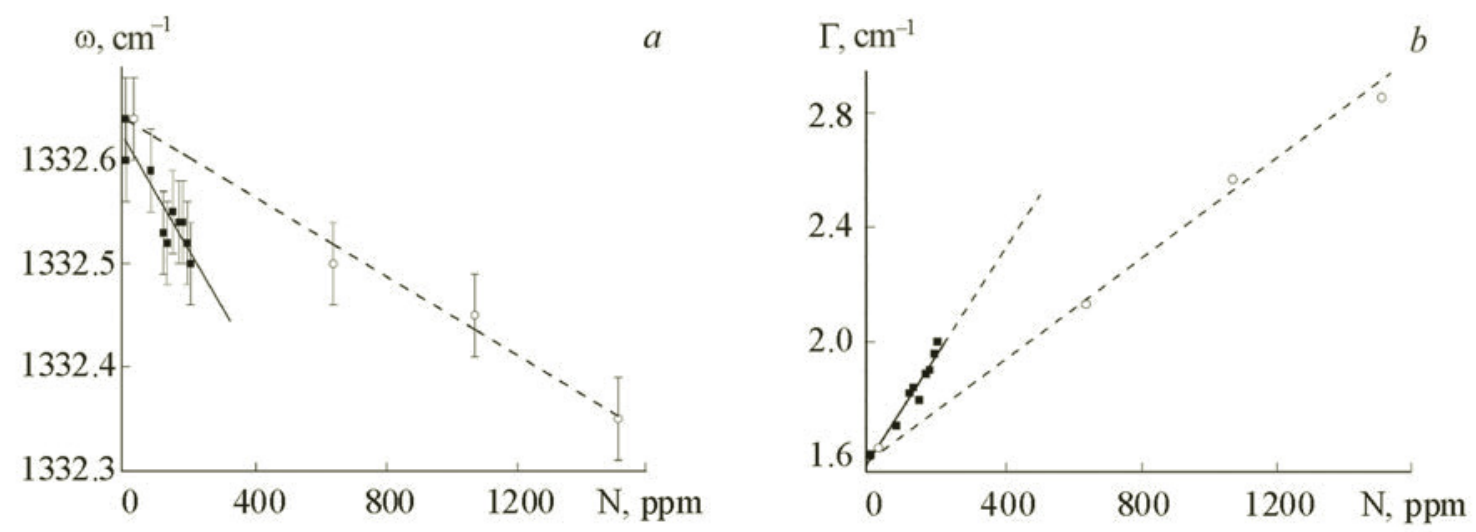

Fig. 4. Position of maximum (a) and half-width (b) of the Raman line on N impurity content in single crystals of synthetic ( $\boldsymbol{\square})$ and natural diamond $(\circ)$; approximation of results using Eq. (5) with $\omega_{0}=1332.2 \mathrm{~cm}^{-1}$ and $k_{i}=1.4 \cdot 10^{-7} \mathrm{ppm}^{-1}$ (solid line) and $\omega_{0}=1332.64 \mathrm{~cm}^{-1}$ and $k_{i}=0.5 \cdot 10^{-7} \mathrm{ppm}^{-1}$ (dashed line) (a); approximation of results using linear functions (dashed lines) (b).

Apparently, different forms of $\mathrm{N}$ impurities in the samples were responsible for the observed differences between natural and synthetic diamonds. Table 1 shows that synthetic diamonds contained the $\mathrm{N}$ impurity as single atoms in substitution positions ( $C$-defect); natural diamonds, polyatomic complexes ( $A$ - and $B 1$-defects). Precise measurements of x-ray diffraction patterns showed that the $\mathrm{N}$ impurity distorted the diamond lattice and increased the unit-cell volume [23-26]. These distortions were proportional to the impurity concentration and also depended on the $\mathrm{N}$ species incorporated into the diamond lattice. In general, the relative change of diamond lattice constant $a$ could be expressed as

$$
\Delta a / a=k_{i} \mathrm{~N}_{i}
$$

where $\mathrm{N}_{i}$ is the content of a particular $\mathrm{N}$-dopant defect (ppm); $k_{i}$, the corresponding proportionality coefficient. According to the literature $[24,25], k_{C}=1.4 \cdot 10^{-7} \mathrm{ppm}^{-1}$ for $C$-defects. The values of $k_{A}$ for $A$-defects vary according to various researchers and span the range from $0.88 \cdot 10^{-7}$ [23] to $0.36 \cdot 10^{-7} \mathrm{ppm}^{-1}$ [26]. Thus, single $\mathrm{N}$ atoms in substitution positions distorted the diamond crystal lattice much more than $\mathrm{N}$ complexes for identical impurity atom concentrations.

Therefore, the dependence of the position of the diamond RS basic line maximum on the impurity concentration should obey the well-known Gruneisen law, according to which the relative change of lattice vibrational frequency is directly proportional to the relative volume change of the unit cell:

$$
-(\Delta v / v)(V / \Delta V)=-d(\log v) / d(\log V)=\gamma
$$

where $v$ is the lattice vibrational frequency; $V$, unit-cell volume; $\gamma$, a constant (Gruneisen parameter). The literature values for the diamond Gruneisen parameter that were obtained by different methods fell in the range 0.9-1.3 [27]. In our opinion, values where the Gruneisen parameter $\Gamma$ was obtained from sequential measurements of RS and x-ray diffraction patterns from the same sample subjected to three-dimensional high-pressure compression are preferred [28, 29]. The value was $\gamma_{i}=0.965 \pm 0.005$ and did not depend on the unit-cell volume for relative densities 1-1.075 [28, 29]. Based on the obtained experimental results and considering the small change of unit-cell volume, the Gruneisen law for the diamond lattice was written as follows

$$
v / v_{0}=\left(V_{0} / V\right)^{0.965}
$$

and showed that Eq. (3) described very well the shift of the RS line maximum with hydrostatic compression in the range 0-35 GPa.

The unit-cell volume change could be expressed in terms of the unit-cell lattice constant:

$$
V_{0} / V=a^{3} /(a+\Delta a)^{3} \sim 1 /(1+3 \Delta a / a) .
$$

Hence, Eq. (3) could be written

$$
\omega=\omega_{0} /(1+3 \Delta a / a)^{0.965}=\omega_{0} /\left(1+3 k_{i} \mathrm{~N}_{i}\right)^{0.965} .
$$


Our experimental results were processed using Eq. (5). Figure 4a shows the results of the calculations. It can be seen that the experimental data agreed well with the predictions made using Eq. (5) with $\omega_{0}=1332.62 \mathrm{~cm}^{-1}$ and $k_{i}=1.4 \cdot 10^{-7} \mathrm{ppm}^{-1}$ for synthetic diamond crystals and $\omega_{0}=1332.64 \mathrm{~cm}^{-1}$ and $k_{i}=0.5 \cdot 10^{-7} \mathrm{ppm}^{-1}$ for natural diamond crystals. The obtained $k_{i}$ values for synthetic and natural diamond crystals agreed well with the literature values for $C$ - and $A$-defects.

Figure 4a shows that in general N-dopant had a weak effect on the position of the diamond RS basic line maximum. Linear functions of the following type could be used to a first approximation to describe the line shift because of the small changes

$$
\omega=\omega_{0}^{T}-q_{i} \mathrm{~N}_{i},
$$

where $\omega_{0}^{T}$ is the position of the line maximum for a structurally perfect crystal at the measurement temperature; $\mathrm{N}_{i}$, concentration of $\mathrm{N}$-dopant as the $i$ th defect; and $q_{i}$, proportionality coefficients. According to the experimental data, $q_{i}=5.5 \cdot 10^{-4}$ and $1.9 \cdot 10^{-4} \mathrm{~cm}^{-1} / \mathrm{ppm}$ for $C$ - and $A$-defects.

The dependences of the diamond RS basic line half-width on N-dopant concentration that were obtained by us for different types of crystals could be written as the linear function

$$
\Gamma=\Gamma_{0}^{T}+f_{i} \mathrm{~N}_{i},
$$

where $\Gamma_{0}^{T}$ is the line half-width for a structurally perfect crystal at the measurement temperature; $f_{i}$, a proportionality coefficient. According to the experimental data, $f_{i}=1.85 \cdot 10^{-3}$ and $0.84 \cdot 10^{-3} \mathrm{~cm}^{-1} / \mathrm{ppm}$ for $C$ - and $A$-defects.

Conclusions. The results showed that the positions of the maxima and half-widths of RS basic lines coincided for structurally perfect natural and synthetic diamond crystals with low dopant contents. Increasing the N-dopant content in the crystals broadened the RS band and shifted its maximum to lower frequency. The magnitude of the observed effect was directly proportional to the concentration of dopant atoms and depended on the form of $\mathrm{N}$-dopant incorporated into the diamond lattice. The change of the diamond RS line parameters with increasing N-dopant content in the crystal agreed well with the magnitude of the crystal-lattice distortions caused by the impurity defects and could be described by the Gruneisen law. In general, the N-dopant had an extremely weak effect on the position of the diamond RS line maximum. Thus, the greatest line shift measured by us was $0.35 \mathrm{~cm}^{-1}$ for natural diamond crystal containing $\sim 1500 \mathrm{ppm}$ of N-dopant as $A$ - and $B 1$-defects. Linear functions with proportionality coefficients $q_{i}=5.5$ and $1.9 \cdot 10^{-4} \mathrm{~cm}^{-1} / \mathrm{ppm}$ for $C$ - and $A$-defects could be used to a first approximation to determine the effect of $\mathrm{N}$-dopant on the position of the RS basic line maximum. The N-dopant had a slightly greater effect on the RS line half-width. The RS line broadening was $\sim 1.2 \mathrm{~cm}^{-1}$ for a natural diamond crystal with $1500 \mathrm{ppm}$ of N-dopant. The obtained functions for the half-width of the diamond RS basic line on the $\mathrm{N}$-dopant concentration for various types of crystals could be described using linear functions with proportionality coefficients $f_{i}=1.85 \cdot 10^{-3}$ and $0.84 \cdot 10^{-3} \mathrm{~cm}^{-1} / \mathrm{ppm}$ for $C$ - and $A$-defects.

\section{REFERENCES}

1. L. Pan and D. Kania, Diamond: Electronic Properties and Applications, Kluwer Acad. Publ., Boston (1995).

2. R. S. Sussmann, CVD Diamond for Electronic Devices and Sensors, Wiley Series Materials for Electronic \& Optoelectronic Applications, New York (2009).

3. Q. Liang, C.-S. Yan, Y. Meng, J. Lai, S. Krasnicki, H.-K. Mao, and R. J. Hemley, Diamond Relat. Mater., 18, 698-703 (2009).

4. Q. Liang, C.-S. Yan, J. Lai, Y.-F. Meng, S. Krasnicki, H. Shu, H.-K. Mao, and R. J. Hemley, Cryst. Growth Des., 14, 3234 3238 (2014).

5. A. M. Zaitsev, Optical Properties of Diamond: A Data Handbook, Springer, Berlin (2001).

6. S. Prawer and R. J. Nemanich, Phil. Trans. R. Soc. London, Ser. A, 362, 2537-2565 (2004).

7. G. Faggio, G. Messina, S. Santangelo, G. Prestopino, I. Ciancaglioni, and M. Marinelli, J. Quant. Spectrosc. Radiat. Transfer, 113, 2476-2481 (2012).

8. K. Ushizawa, M. Nishitani-Gamo, K. Watanabe, I. Sakaguchi, Y. Sato, and T. Ando, J. Raman Spectrosc., 30, 957-961 (1999).

9. P. Gonon, E. Gheeraert, A. Deneuville, F. Fontaine, L. Abello, and G. Lucazeau, J. Appl. Phys., 78, 7059-7062 (1995).

10. M. Bernard, A. Deneuville, and P. Muret, Diamond Relat. Mater., 13, 282-286 (2004).

11. S. A. Solin and A.K. Ramdas, Phys. Rev. B: Solid State, 1, 1687-1698 (1970). 
12. G. A. Gusakov, M. P. Samtsov, E. S. Voropai, V. S. Solov'ev, and A. N. Demenshchenok, Zh. Prikl. Spektrosk., 68, No. 5, 612-616 (2001) [G. A. Gusakov, M. P. Samtsov, E. S. Voropai, V. S. Solov'ev, and A. N. Demenshchenok, J. Appl. Spectrosc., 68, 799-805 (2001)].

13. H. Hanzawa, N. Umemura, Y. Nisida, H. Kanda, M. Okada, and M. Kobayashi, Phys. Rev. B: Condens. Matter Mater. Phys., 54, 3793-3799 (1996).

14. N. V. Surovtsev, I. N. Kupriyanov, V. K. Malinovsky, V. A. Gusev, and Yu. N. Pal'yanov, J. Phys.: Condens. Matter, 11, 4767-4774 (1999).

15. T. A. Nachal'naya, V. D. Andreyev, and E. V. Gabrusenok, Diamond Relat. Mater, 3, 1325-1328 (1994).

16. G.-F. Huang, X. P. Jia, Y. Li, M.-H. Hu, Z.-C. Li, B.-M. Yan, and H.-A. Ma, Chin. Phys. B, 20, 078103 (2011).

17. G. A. Gusakov, M. P. Samtsov, and E. S. Voropai, Zh. Prikl. Spektrosk., 84, No. 4, 545-553 (2017) [G. A. Gusakov, M. P. Samtsov, and E. S. Voropai, J. Appl. Spectrosc., 84, 573-580 (2017)].

18. Y. N. Palyanov, I. Yu. Malinovskii, Yu. M. Borzdov, A. F. Khokhryakov, A. I. Chepurov, A. A. Godovikov, and N. V. Sobolev, Dokl. Akad. Nauk SSSR, 315, No. 5, 1221-1224 (1990).

19. Y. N. Palyanov, Y. M. Borzdov, A. F. Khokhryakov, I. N. Kupriyanov, and A. G. Sokol, Cryst. Growth Des., 10, 3169-3175 (2010).

20. T. D. Ositinskaya, V. A. Tsendrovskii, and A. S. Vishnevskii, Inzh. Fiz. Zh., 32, No. 4, 620-640 (1977).

21. C. A. Parker, Photoluminescence of Solutions with Applications to Photochemistry and Analytical Chemistry, Elsevier, New York (1968), 544 pp.

22. M. J. Lipp, V. G. Baonza, W. J. Evans, and H. E. Lorenzana, Phys. Rev. B: Condens. Matter Mater. Phys., 56, 5978-5984 (1997).

23. W. Kaiser and W. L. Bond, Phys. Rev., 115, 857-863 (1959).

24. A. R. Lang, M. Moore, A. P. W. Makepeace, W. Wierzchowski, and C. M. Welbourn, Phil. Trans. R. Soc. London, Ser. A, 337, 497-520 (1991).

25. T. Sato, K. Ohashi, T. Sudoh, K. Haruna, and H. Maeta, Phys. Rev. B: Condens. Matter Mater. Phys., 65, 092102(R) (2002).

26. A. R. Lang and G. Pang, Phil. Trans. R. Soc. London, Ser. A, 356, 1397-1419 (1998).

27. S. S. Mitra, O. Brafman, W. B. Daniels, and R. K. Crawford, Phys. Rev., 186, 942-944 (1969).

28. I. V. Aleksandrov, A. F. Goncharov, and S. M. Stishov, Pis'ma Zh. Eksp. Teor. Fiz., 44, 474-476 (1986).

29. I. V. Aleksandrov, A. F. Goncharov, A. N. Zisman, and S. M. Stishov, Zh. Eksp. Teor. Fiz., 93, 680-691 (1987). 